\title{
Case Detection Testing for Primary Aldosteronism in Male Patients with Hypertension and Snoring
}

Menghui Wang ${ }^{1}$, Nanfang Li ${ }^{* 1}$, Ying Zhang ${ }^{1}$, Delian Zhang ${ }^{1}$, Suofeiya Abulikemu ${ }^{1}$, Yinchun Wang ${ }^{1}$, Guli Nuer ${ }^{1}$, Jianqiong Kong ${ }^{1}$, Juhong

Zhang $^{1}$, Zhitao Yan ${ }^{1}$, Hongjian Li ${ }^{1}$, and Xiangyang Zhang ${ }^{2}$

${ }^{1}$ Hypertension Institute of Xinjiang; Hypertension Center of the People's Hospital Of Xinjiang Uygur Autonomous Region, PR China

${ }^{2}$ The First Affiliated Hospital of Xinjiang Medical University, 830000, Urumqi, PR China

\begin{abstract}
Objectives: Coexistence of primary aldosteronism and obstructive sleep apnea in hypertension is evidenced. However, aldosterone and renin activity is varying with apnea/hypopnea index changes in subjects with resistant hypertension. Thus, the aim is to investigate the optional cutoff value for aldosterone/renin activity to screen primary aldosteronism in patients with different status of apnea/hypopnea index.

Methods: 271 hypertensive male snores were evaluated via polysomnography and divided into two groups, group with apnea/hypopnea index $>15$ events/h and with apnea/hypopnea index $<15$ events/h. Primary aldosteronism was determined as following: subjects with plasma renin activity $<1.0 \mathrm{ng} / \mathrm{mL} / \mathrm{h}$ and aldosterone concentration>15 $\mathrm{ng} / \mathrm{dL}$ performed saline infusion test, after which aldosterone concentration $>5 \mathrm{ng} / \mathrm{dL}$ was a sign of primary aldosteronism. Receiver operating characteristic curve was applied to explore appropriate cutoff value for aldosterone/renin activity.

Results: $39(14.4 \%)$ of the 271 were diagnosed with primary aldosteronism including 15 with apnea/hypopnea index $<15$ events/h and 24 with apnea/hypopnea index $>15$ events/h. Area under receiver operating characteristic curve was $0.97(95 \% \mathrm{Cl} 0.94-0.99)$ in the group with apnea/hypopnea index $>15$ events/h and $0.91(95 \% \mathrm{Cl} 0.87-0.96)$ in the group with apnea/hypopnea index $<15$ events/h. Appropriate cutoff value for aldosterone/renin activity is $29.1 \mathrm{ng} / \mathrm{dL}$ per $\mathrm{ng} / \mathrm{mL} / \mathrm{h}$ for the group with apnea/hypopnea index $<15$ events/h with sensitivity $100 \%$, specificity $90 \%$ and $15.9 \mathrm{ng} / \mathrm{dL}$ per $\mathrm{ng} / \mathrm{mL} / \mathrm{h}$ for the group with apnea/hypopnea index $>15$ events/h with sensitivity $100 \%$, specificity $69.7 \%$. Youden index is 0.9 for the group with apnea/hypopnea index $<15$ events/h and 0.7 for the group with apnea/hypopnea index $>15$ events/h.
\end{abstract}

Conclusions: Optional cutoff values of aldosterone/renin activity to screening for primary aldosteronism should be considered in patients with different status of apnea/hypopnea index.

Keywords: Obstructive sleep apnea; Primary aldosteronism; Aldosterone/renin activity ratio; Receiver-operating-characteristics curves

\section{Abbreviations}

AHI: Apnea-Hypopnea Index, events/h; ARR: Aldosterone/Renin Activity, ng/dL per ng/mL/h; AUC: Area Under the receiver operating characteristic Curve; BMI: Body Mass Index, kg/m²; BP: Blood Pressure; DBP: Diastolic Blood Pressure, $\mathrm{mmHg}$; NPV: Negative Predictive Value; PA: Primary Aldosteronism; PAC: Plasma Aldosterone Concentration, ng/dL; PPV: Positive Predictive Value; PRA: Plasma Renin Activity, ng/mL; ROC: Receiver Operating characteristic Curve; SBP: Systolic Blood Pressure, mmHg; SIT: Saline Infusion Test; WC: Waist Circumference, $\mathrm{cm}$.

\section{Introduction}

Overall the prevalence of hypertension appears to be around 30$40 \%$ of the general population. The relationship between blood pressure (BP) values and cardiovascular and events has been addressed in a body of observational studies [1,2]. Etiology of hypertension is manifold and complicated, involving life styles of individuals, environmental factors, secondary diseases and gene polymorphisms [3-7]. Snoring is a common phenomenon. Identified obstructive sleep apnea (OSA) via polysomnography appears to be the most common condition association with resistant hypertension [8]. OSA affects hypertensive patients through other cardiovascular diseases, including coronary artery disease, arrhythmia and stroke [9-11].

Primary aldosteronism (PA) is a well-known endocrine cause of hypertension in which aldosterone production is inappropriately high, causing suppression of plasma renin, hypertension, sodium retention and urinary potassium excretion. It is important to identify PA is not only because it contains higher prevalence, affecting $5-18 \%$ of hypertensive patients with potentially curable condition, but also because that PA plays a key role in cardiovascular disease states beyond hypertension. Since the recommendation of the aldosterone/renin activity (ARR) as a screening tool for PA, there has been a marked increase prevalence of PA in different selected populations [12-17].

Evidences have provided by a case report that the coexistence presences in patients with PA and OSA [18-20]. It is also necessary to screen PA in patients with hypertension and scorning in order to obtain an opportunity of specific treatments. However, renin-angiotensinaldosterone system (RAAS) and sympathetic nervous system (SNS)

*Corresponding author: Nanfang Li, Hypertension Institute of Xinjiang, Hypertension Center of the People's Hospital of Xinjiang Uygur Autonomous Region, 91 Tianchi Road, Urumqi City, Xinjiang Province 830054, PR China, Tel: 86-13999179937; Fax: 86-9918564816; E-mail: Inanfang2010@sina.com

Received August 28, 2014; Accepted September 29, 2014; Published October 01,2014

Citation: Wang M, Nanfang Li, Zhang Y, Zhang D, Abulikemu S, et al. (2014) Case Detection Testing for Primary Aldosteronism in Male Patients with Hypertension and Snoring. J Hypertens 3: 180. doi:10.4172/2167-1095.1000180

Copyright: ( 2014 Wang M, et al. This is an open-access article distributed under the terms of the Creative Commons Attribution License, which permits unrestricted use, distribution, and reproduction in any medium, provided the original author and source are credited. 
are activated by recurrent hypoxia and periodic arousals from sleep in OSA [21-24]. Meanwhile, it has been shown than aldosterone and renin activity changes with apnea/hypopnea index (AHI) severity in subjects with resistant hypertension, suggesting that ARR might bear some variability in various degrees of OSA [25]. Thereby, we speculated that cutoff value for ARR in patients with snoring is not standardized due to potential changes in aldosterone and renin activity with several of severity of OSA. Thus, aim of the current study is to investigate the optional cutoff value for ARR to screen PA in subjects with various severity of OSA.

\section{Methods}

\section{Patients}

Some male patients with hypertension were initially referred to the Center of Diagnosis, Treatment and Research of Hypertension in Xinjiang between August 2009 and July 2010. The histories of snoring and smoking have been confirmed by physicians' inquiry. All subjects' symptoms of sleep apnea were made clear by patients themselves or their family's members. And they were advised to discontinue diuretics (including spironolactone) for 6 weeks, and $\beta$-blockers, angiotensin-converting enzyme inhibitors and angiotensin receptor blockers for 4 weeks, and to have a two weeks follow-up. If antihypertensive medication could not be withdrawn completely, patients were prescribed a-blockers (doxazosin mesylate controlledrelease; $2-8 \mathrm{mg} /$ day or terazosin hydrochloride, $2-10 \mathrm{mg} /$ day) and/ or non-dihydropyridine calcium channel blockers (verapamil slowrelease, $120-480 \mathrm{mg} /$ day) for at least $4-6$ weeks before screening. The drug dose depended on blood pressure (BP) level and drug tolerability in individual patients. The exclusion criteria were: (i) confounding conditions such as acute upper respiratory infection, a history of chronic obstructive pulmonary disease, asthma, congestive heart failure, arrhythmia, angina pectoris, serum creatinine $>177 \mathrm{mmol} / \mathrm{L}$, pregnancy/breastfeeding; or (ii) use of drugs that might interfere with the renin-aldosterone axis (e.g., corticosteroids, sex hormones, licorice, or nonsteroidal anti-inflammatory drugs). All of these works were finished in out-patients. Finally, 271 patients were admitted to the hospital to investigate the etiology of hypertension. Demographic parameters were noted in all patients groups: body mass index (BMI), waist circumference (WC) and clinic blood pressure.

\section{Hormone assays}

Blood samples were stored in an ice bath at $4^{\circ} \mathrm{C}$ during transportation. Blood tubes were then centrifuged in a refrigerated centrifuge, and plasma was separated from the cells immediately after centrifugation. The plasma samples were divided into two parts: one part was used for the determination of plasma Ang I (AIa)and the other part was used for the determination of plasma $\mathrm{AIb}$ concentrations following 1 -h incubation at $37^{\circ} \mathrm{C}$ followed by reacting the sample with direct antibodies [26]. Plasma renin activity (PRA) was calculated using the following formula: (AIb-AIa)/hour. AngI was measured by radioimmunoassay (Center of Beifang Biology Technique, Beijing, China). The reference range for PRA was $0.20-1.90 \mathrm{ng} / \mathrm{mL} / \mathrm{h}$. For the kit, the intra- and inter-assay coefficients of variation were $\leq 10-15 \%$. PAC was also measured by radioimmunoassay (DSL-8600ACTIVE Aldosterone Coated-Tube Radioimmunoassay Kit; Diagnostic Systems Laboratories, Webster, TX, USA). The reference range for PAC was $3.37-23.74 \mathrm{ng} / \mathrm{dL}$. The intra- and inter-assay coefficients of variation were within the range $4.5-9.8 \%$.

\section{Collection of 24-h urine and blood samples to measure potassium levels}

Twenty-four-hour urine collection was performed according to standard procedures before Saline Infusion Test (SIT). Patients were on a regular diet without vitamins or mineral supplements. Urine was collected continuously from the first bladder void at 7 am on the first day until 7 am on the second day. The collection jar was stored in a refrigerator at $4^{\circ} \mathrm{C}$. After $24 \mathrm{hrs}$, the total urine volume was measured and a 15-ml urine sample was saved to measure the level of $\mathrm{K}^{+}$. Venous blood samples were taken simultaneously to measure serum $\mathrm{K}^{+}$on the second morning after a 12 -h fast. No tourniquet was used and no fist was made during blood collection. Blood was drawn slowly using a syringe and then transferred into a non-vacuum tube for immediate measurement of serum $\mathrm{K}^{+}$. Hypokalemia was defined as serum $\mathrm{K}^{+}$ $<3.50 \mathrm{mmol} / \mathrm{L}$.

\section{Laboratory assessment}

Serum $\mathrm{K}^{+}$, urinary $\mathrm{K}^{+}$and other biochemical content were measured by enzymatic methods using an auto-analyzer (7600-010 Automatic Analyzer; Hitachi Medical Systems, Suzhou, China). All blood samples were examined within 1 day of collection at the Hypertension Clinical Research Center of the People's Hospital of Xinjiang Uygur Autonomous Region.

All patients gave their informed consent after a detailed description of the experimental protocol, which was approved by the local Ethical Committee.

\section{Screening and diagnosis of OSA}

271 male patients with hypertension and snoring underwent fullnight, diagnostic polysomnography. Polysomnographic evaluation included airflow monitoring with thermocouple; respiratory effort with belts at the chest and abdominal positions; oxygen saturation measured by pulse oximetry; heart rate with a single-lead ECG; EEG (C4-A1, C3-A2); submental and tibial electromyograms and bilateral electrooculograms. Apnea was defined as a cessation in airflow for $\geq 10$ s. Hypopnea was defined as $>50 \%$ reduction in respiratory flow or $>30 \%$ reduction linked to more than $3 \%$ desaturation and/or microarousals at least. AHI was calculated as the total number of apneas plus hypopneas divided by the hours of sleep. OSA was defined as an AHI $>5$ events $/ \mathrm{h}$, and further subclassified into mild OSA (AHI=5-15), moderate OSA $(\mathrm{AHI}=16-30)$, and severe OSA (AHI $>30)$. A habitual snorer is a subject who always snores at night, while the AHI is $<5$ events/h [27].

\section{The diagnosis protocol of PA}

\section{Screening test: PRA, PAC and ARR}

i. We used the screening criteria described as $\mathrm{PRA}<1.0 \mathrm{ng} / \mathrm{mL} / \mathrm{h}$ and PAC $>15 \mathrm{ng} / \mathrm{dL}$ [28].

ii. The ARR was calculated using PAC as the numerator and PRA as the denominator, and expressed in $\mathrm{ng} / \mathrm{dL}$ per $\mathrm{ng} / \mathrm{mL} / \mathrm{h}$. In samples with a PRA $<0.2 \mathrm{ng} / \mathrm{mL} / \mathrm{h}$ (i.e., primarily corresponding to subjects with a final diagnosis of PA), a PRA value of $0.2 \mathrm{ng} / \mathrm{mL} / \mathrm{h}$ was used to calculate the ARR.

Confirmatory test for PA: saline infusion test: Subjects were asked to sit for $30 \mathrm{~min}$ at $11 \mathrm{am}$, followed by blood-sample collection for measurements of PAC and PRA. Blood samples were drawn after an infusion of $2 \mathrm{~L}$ of $0.9 \%$ saline over $4 \mathrm{~h}$. The SIT should not be performed in patients with severe uncontrolled hypertension or 
hypokalemia. Serum $\mathrm{K}^{+}$levels were brought within the normal range (3.5-5.5 mmol/ $/ \mathrm{L} \mathrm{K}^{+}$) with oral $\mathrm{K}^{+}$supplement before the test in patients with hypokalemia. This test was preformed after collecting 24 - $\mathrm{h}$ urine and blood samples to measure serum $\mathrm{K}^{+}$levels. Post-infusion $\mathrm{PAC}<5$ $\mathrm{ng} / \mathrm{dL}$ made a diagnosis of PA unlikely, and levels $>5 \mathrm{ng} / \mathrm{dL}$ were a very probable sign of PA [29] (the protocol can be seen in Table 3).

\section{Statistics analysis}

Data are given as mean \pm SD or percentage. Differences in dose data between groups were assessed using Student's t-tests or $\chi^{2}$ test where appropriate.

ROC analysis was used to evaluate the power of screening for PA using different ARR levels between two groups with AHI $>15$ and $\mathrm{AHI}<15$ events/h. Sensitivity (i.e. true positive rate) and specificity (i.e. true negative rate) were calculated for each ARR cut-off value. A ROC curve that plotted sensitivity against (1-specificity) was drawn. Youden's index is easily calculated according to formula $J=$ sensitivity + specificity -1. Data analyses were carried out using SPSS for Windows (version 17.0; SPSS, Chicago, IL, USA). Two-tailed P values are provided for descriptive purposes, with $P<0.05$ considered statistically significant.

\section{Results}

In total, 271 male subjects with hypertension were evaluated (Table 1). In 227 (83.3\%) of 271 male patients with hypertension who snore regularly were diagnosed to have OSA via PSG presenting AHI more than or equal to 5 event/h, and of those 227 confirmed to have OSA, 131 (57.6\%) presented AHI $>15$ events/h. PA was diagnosed in 39 (14.4\%) patients: 15 (38.4\%) of patients with $\mathrm{AHI}<15$ events/h and $24(61.6 \%)$ of patients with AHT>15 events/h 39 (14.4\%) on the basis of diagnostic schema for PA (Table 3). Of those with AHI more than or equal to 5 events/h, 32 patients were made a diagnosis of PA, accounting for $11.8 \%$. Mean age of participants enrolled was $46 \pm 9$ years (range 19-71 years), mean abdominal circumference was $100.8 \pm$ $9.8 \mathrm{~cm}$, and mean BMI was $28.6 \pm 65 \mathrm{~kg} / \mathrm{m}^{2} .26$ patients were confirmed to have hypokalemia, accounting for $17.4 \%$. There were no significant difference in age, BMI, SBP, DBP, serum potassium, PRA, PAC and ARR ( $p>0.05$ for all) between AHI $>15$ events/h group and $\mathrm{AHI}<15$ events/h group, but waist circumference and urinary potassium were significantly higher in the group with $\mathrm{AHI}>15$ events/h than in the group with $\mathrm{AHI}<15$ events/h ( $p<0.05$ for the two).
ROC curve was applied to explore the appropriate cut off value for screening of PA in the two groups with AHI $>15$ events/h and $\mathrm{AHI}<15$ events/h. Area under ROC was 0.97 (95\% CI 0.94-0.99) in the group with AHI $>15$ (Figure 1) and 0.91 (95\% CI 0.87-0.96) in the group with $\mathrm{AHI}<15$ events/h (Figure 2). Table 2 shows that the most appropriate cutoff value for ARR to screen PA is $29.1 \mathrm{ng} / \mathrm{dL}$ per $\mathrm{ng} / \mathrm{mL} / \mathrm{h}$ for the group with $\mathrm{AHI}<15$ events/h with sensitivity of $100 \%$, specificity of $90 \%$, positive predictive value of $60.0 \%$, and negative predictive value of $100 \%$ and $15.9 \mathrm{ng} / \mathrm{dL}$ per $\mathrm{ng} / \mathrm{mL} / \mathrm{h}$ for the group with $\mathrm{AHI}>15$ events $/ \mathrm{h}$ with sensitivity of $100 \%$ and specificity of $69.7 \%$ positive predictive value of $37.5 \%$, and negative predictive value of $100 \%$. Youden index is 0.9 for the group with $\mathrm{AHI}<15$ events/h and 0.7 for the group with $\mathrm{AHI}$ $>15$ events/h, respectively.

Table 3 presents the prevalence of $\mathrm{PA}$ in patients with $15.9<$ ARR $<29.1 \mathrm{ng} / \mathrm{dL}$ per $\mathrm{ng} / \mathrm{mL} / \mathrm{h}$ on the basis of diagnostic schema. PA was not found in patients with $\mathrm{AHI}<15$ events/h who had $15.9<\mathrm{ARR}<29.1 \mathrm{ng} / \mathrm{dL}$ per $\mathrm{ng} / \mathrm{mL} / \mathrm{h}$, but 4 patients with PA (19\%) were confirmed who had AHI $>15$ events/h and $15.9<\mathrm{ARR}<29.1 \mathrm{ng} / \mathrm{dL}$ per $\mathrm{ng} / \mathrm{mL} / \mathrm{h}$.

\section{Discussion}

In our study, we found two important results. The different cutoff values of ARR indeed exist in patients with different levels of AHI. Appropriate cutoff value for ARR is $29.1 \mathrm{ng} / \mathrm{dL}$ per $\mathrm{ng} / \mathrm{mL} / \mathrm{h}$ for the group with $\mathrm{AHI}<15$ events/h and $15.9 \mathrm{ng} / \mathrm{dL}$ per $\mathrm{ng} / \mathrm{mL} / \mathrm{h}$ for the group with AHI $>15$ events/h. While researched population has been limited in male hypertensive patients with snoring complained by themselves or their family members.

Obstructive sleep apnea and PA have been considered the most common form of secondary hypertension with potentiality if managed systemically and specifically. Coexistence of the two prompted researchers to investigate potential mechanisms related to OSA and PA. Animal model have evidenced that aldosterone acts as the central driver to increase activity of brain renin angiotension system, oxidative stress and sympathetic nervous system [30], which may disregulate central breathing system. In addition, aldosterone excess secondary to RAAS activation may also contribute to OSA through increased edema at nasopharyngeal tissues. It has been indicated in a body of studies that increased sodium and water retention, secondary to

\begin{tabular}{|c|c|c|c|c|c|c|c|}
\hline & \multicolumn{2}{|c|}{ Total } & \multicolumn{3}{|c|}{$\mathrm{AHI}<15$} & \multicolumn{2}{|l|}{$\mathrm{AHI}>15$} \\
\hline & $\mathrm{n}$ & value & $\mathrm{n}$ & value & $\mathrm{n}$ & Value & $p$ \\
\hline Age & 271 & $44(8)$ & 115 & $43(9)$ & 156 & $45(8)$ & n.s \\
\hline WC & 263 & $100.8(9.8)$ & 111 & $98.9(11.3)$ & 152 & $102.2(8.3)$ & $<0.05$ \\
\hline BMI & 261 & $28.6(6.5)$ & 111 & $28.1(9.3)$ & 150 & $28.9(3.1)$ & n.s \\
\hline Smoke & 271 & $171(63.1 \%)$ & 115 & $72(62.6 \%)$ & 156 & $99(63.5 \%)$ & n.s \\
\hline SBP & 269 & $142(22)$ & 115 & $141(23)$ & 154 & $143(20)$ & n.s \\
\hline DBP & 269 & $99(14)$ & 115 & $98(14)$ & 154 & $99(14)$ & n.s \\
\hline Serum $\mathrm{K}^{+}$ & 264 & $3.82(0.36)$ & 111 & $3.83(0.37)$ & 153 & $3.82(0.35)$ & n.s \\
\hline Urinary $\mathrm{K}^{+}$ & 246 & $42.1(17.1)$ & 109 & $39.3(14.2)$ & 137 & $44.3(18.9)$ & $<0.05$ \\
\hline PRA & 271 & $1.77(1.69)$ & 115 & $1.96(1.93)$ & 156 & $1.62(1.47)$ & n.s \\
\hline PAC & 271 & $15.12(7.24)$ & 115 & $14.55(7.40)$ & 156 & $15,54(7.11)$ & n.s \\
\hline ARR & 271 & $28.4(49.7)$ & 115 & $18.40(22.22)$ & 156 & $21.78(24.38)$ & n.s \\
\hline $\mathrm{AHI}$ & 271 & $25.2(22.4)$ & 115 & $6.62(4.29)$ & 156 & $38.88(20.31)$ & $<0.0001$ \\
\hline PA & 271 & $39(14.4 \%)$ & 115 & $15(13.0 \%)$ & 156 & $24(15.4 \%)$ & n.s \\
\hline
\end{tabular}

Data are shown as mean \pm SD, except smoke and PA, which are percentages.

AHI: Apnea-Hypopnea Index; events/h, ARR: Aldosterone/Renin Activity; ng/dL per ng/mL/h; BMl: Body Mass Index; kg/m2; DBP: Diastolic Blood Pressure: $\mathrm{mmHg}$; PA: Primary Aldosteronism; PAC: Plasma Aldosterone Concentration; ng/dL; PRA: Plasma Renin Activity; ng/mL; SBP: Systolic Blood Pressure; mmHg; WC: Waist Circumference; $\mathrm{cm}$

Table 1: Demographic and biochemical values for all of the subjects and for subjects according to apnea-hypopnea index. 
Citation: Wang M, Nanfang Li, Zhang Y, Zhang D, Abulikemu S, et al. (2014) Case Detection Testing for Primary Aldosteronism in Male Patients with Hypertension and Snoring. J Hypertens 3: 180. doi:10.4172/2167-1095.1000180

\begin{tabular}{|l|c|c|}
\hline & \multicolumn{2}{|c|}{ ARR } \\
\hline cut-off values & AHI<15 & AHI>15 \\
\hline AUC & 29.1 & 15.9 \\
\hline Sensitivity (\%) & 0.97 & 0.91 \\
\hline Specificity (\%) & $100.0 \%$ & $100.0 \%$ \\
\hline PPV (\%) & $90.0 \%$ & $69.7 \%$ \\
\hline NPV (\%) & $60.0 \%$ & $37.5 \%$ \\
\hline Youden & $100.0 \%$ & $100.0 \%$ \\
\hline
\end{tabular}

AHI: Apnea-Hypopnea Index; events/h; ARR: Aldosterone/Renin Activity; ng/dL pe $\mathrm{ng} / \mathrm{mL} / \mathrm{h}$; AUC: Area Under the receiver operating characteristic Curve; PPV: Positive Predictive Value; NPV: Negative Predictive Value

Table 2: Diagnostic power of ARR in the different status of apnea-hypopnea index.

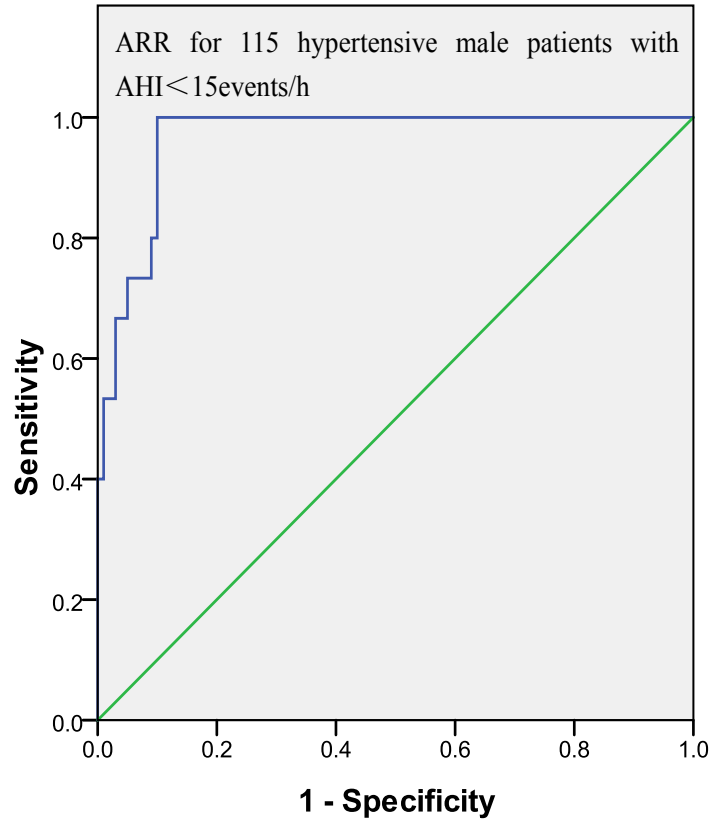

Figure 1: Receiver operating characteristic curve for aldosterone/renin activity in 15 proven primary aldosteronism determined by protocol; area under the receiver operating characteristic curve: $0.97(95 \% \mathrm{Cl} 0.94-0.99)$.

hyperaldosteronism, lead to or worsen OSA [31], even in resistant hypertension [32], and treatment with diuretics improves upper airway caliber and OSA in patients with diastolic heart failure [33], implicating that pharyngeal edema is a possible cause of upper airway collapse during sleep. Meanwhile, displacing fluid from the lower extremities to the neck results in narrowing of upper airway, suggesting that upper airway edema may contribute importantly to the pathogenesis of OSA [34]. Spironolactone, a mineralocorticoid receptor antagonist, has been shown to reduce severity of OSA in patients with resistant hypertension and therefore researchers explained that spironolactone reduces peripharyngeal fluid accumulation and thereby lowers risk of upper airway obstruction by reversing chronic fluid retention, whereas the precise mechanism behind needs to be confirmed in randomized trials with larger samples with resistant hypertension [35], and meanwhile continuous positive airway pressure therapy reportedly reduces elevated aldosterone levels in patients with OSA syndrome, suggesting that the two entities may be causally related, that is, one contributing to the other [36].

Carolina and colleagues noted an association among resistant hypertension, aldosterone and OSA in their serial studies: in patients with resistant hypertension, aldosterone excretion is increased with symptoms of OSA. Furthermore, plasma aldosterone concentration is positively correlated with severity of OSA; on the contrary, severity of OSA is strongly correlated with the degree of urinary aldosterone excess in the high-aldosterone group. Letizia et al. reported, in 53 patients with essential hypertension and OSA, that there is a positive linear correlation between plasma renin activity and AHI. However, our current study observed no significant difference in PRA and PAC between two groups with $\mathrm{AHI}>15$ and $\mathrm{AHI}<15$ events/h. One of the possible reasons is different population selected. In our study, we limited study subjects to male gender with common hypertension and snoring. However, based on our results, the elevation of urinary

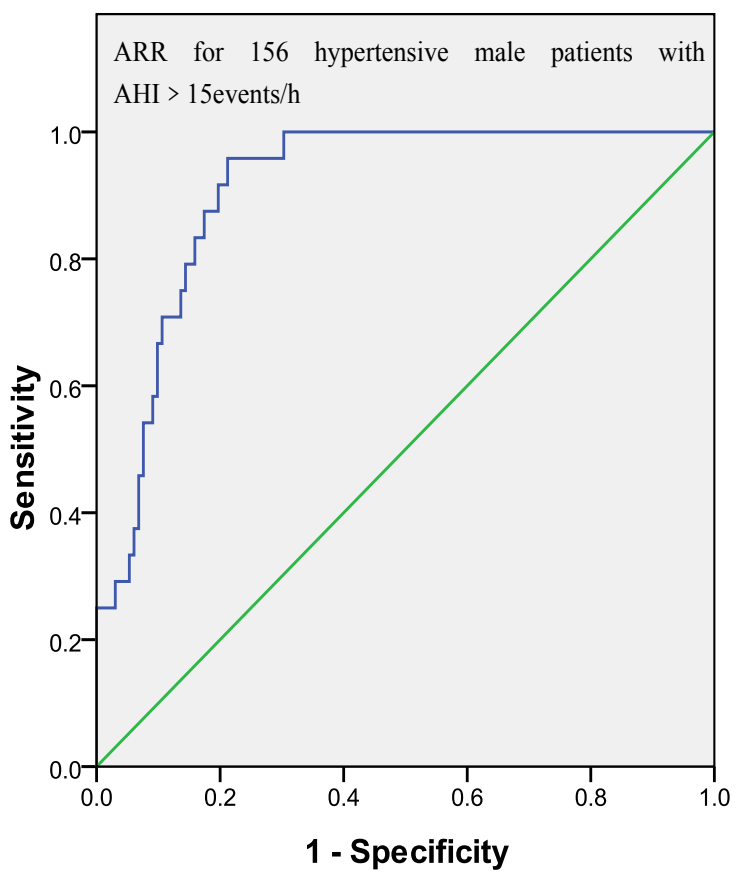

Figure 2: Receiver operating characteristic curve for aldosterone/renin activity in 24 proven primary aldosteronism determined by protocol; area under the receiver operating characteristic curve: $0.91(95 \% \mathrm{Cl} 0.87-0.96)$.

\begin{tabular}{|l|c|c|}
\hline \multirow{2}{*}{$\mathrm{N}(\%)$} & \multicolumn{2}{|c|}{$\mathbf{1 5 . 9 < \text { ARR } < 2 9 . 1}$} \\
\hline$\downarrow$ & AHI<15 & AHI $>15$ \\
\hline $\mathrm{PRA}<1.0$ & $9(100 \%)$ & $21(100 \%)$ \\
\hline $\mathrm{PAC}>15$ & $\downarrow$ & $\downarrow$ \\
\hline$\downarrow$ & $8(88.9 \%)$ & $20(95.2 \%)$ \\
\hline $\mathrm{PRA}<1.0$ and PAC>15 & $1(11.1 \%)$ & $8(30.1 \%)$ \\
\hline$\downarrow$ & $\downarrow$ & $\downarrow$ \\
\hline $\mathrm{SIT}$ & $0(0 \%)$ & $7(33.3 \%)$ \\
\hline$\downarrow$ & $\downarrow$ & $7(33.3 \%)$ \\
\hline $\mathrm{PAC}>5$ after SIT & $0(0 \%)$ & $\downarrow$ \\
\hline$\downarrow$ & $\downarrow$ & $4(19.0 \%)$ \\
\hline $\mathrm{PA}$ & $0(0 \%)$ & $\downarrow$ \\
\hline
\end{tabular}

AHI: Apnea-Hypopnea Index; events/h; ARR: Aldosterone/Renin Activity; ng/dL per ng/mL/h; PA: Primary Aldosteronism; PAC: Plasma Aldosterone Concentration; ng/ dL; PRA: Plasma Renin Activity; ng/mL; SIT: Saline Infusion Test

Table 3: The protocol for diagnosis of primary aldosteronism and prevalence of PA in patients with $15.9<A R R<29.1 \mathrm{ng} / \mathrm{dL}$ per $\mathrm{ng} / \mathrm{mL} / \mathrm{h}$. 
potassium excretion was more manifest in patients with $\mathrm{AHI}>15$ events/h. The confounding factors of increasing kaliuresis include sodium intake, potassium consumption, diuretic, renal dysfunction, and other rare diseases excluding PA. All patients referred in our centre were suggested a low-salt diet although uniform food and drink were not distributed to them. In spite of hypokalemia, potassium supplement was not performed before completing 24-hour urine collection. The confounding effects of diuretics and renal dysfunction have been excluded based on methods of current study. Other diseases leading to increase kaliuresis such as renal tubular acidosis might be ignored to identify, whereas the common causes of this disease were excluded when patients were selected. In addition, the higher WC was found in the group with AHI $>15$ events/h in spite of similarity of BMI between two groups. In obesity, activation of RAAS is increased in an adipose tissue and in circulation [37]. The comparing results between patients with $\mathrm{AHI}>15$ and $<15$ events/h predicted that prevalence of PA might be underestimated in patients with AHI $>15$ events/h. Therefore, we conducted the current study to investigate an optional cutoff value for ARR to screen PA in patients with OSA of various degrees.

ARR is currently the most reliable and available means of screening $\mathrm{PA}$, recommended by current guidelines. Cutoff value of ARR ranging from 20 to $100 \mathrm{ng} / \mathrm{dL}$ per $\mathrm{ng} / \mathrm{mL} / \mathrm{h}$ has been referenced in selected or unselected hypertensive subjects [29,38]. A further result of our study showed that different cutoff values of indeed existed in patients with different AHI. For patients with $\mathrm{AHI}<15$ events/h, misdiagnosis might be less frequent if $\mathrm{ARR}>20 \mathrm{ng} / \mathrm{dL}$ per $\mathrm{ng} / \mathrm{mL} / \mathrm{h}$ used as recommended by guideline. Nevertheless, for patients with AHI $>15$ events/h, it is a more reasonable to use ARR $>20 \mathrm{ng} / \mathrm{dL}$ per $\mathrm{ng} / \mathrm{mL} / \mathrm{h}$ as a cutoff value. Plasma renin and aldosterone levels have usually been found to rise steadily at onset of sleep and reach their highest levels during the second half of the night. The relationship between RAAS and OSA is complex and remains to be elucidated. Activation of RAAS by recurrent hypoxia and arousal may contribute to elevation of blood pressure in OSA. Elevated PRA probably cause a declination of ARR. Consequently, reassessment of cutoff value for ARR to screen for PA should be considered.

In general, higher ARR is meaningful only when PAC exceeds 15 $\mathrm{ng} / \mathrm{dL}$. In order to evaluate optional cutoff value of ARR in patients with snoring, the protocol was performed without involving ARR, whereas using $\mathrm{PRA}<1.0 \mathrm{ng} / \mathrm{mL} / \mathrm{h}$ and $\mathrm{PAC}>15 \mathrm{ng} / \mathrm{dL}$ as an initial screening criteria. Although this criterion was not recommended by the guideline, it might be more in accordance with the definition of PA. Just few studies investigated the relationship between some indicators about PA and OSA, while most studies involved in primary hyperaldosteronism due to lacking of suppression tests. Primary hyperaldosteronism was defined as PRA $<1.0 \mathrm{ng} / \mathrm{mL} / \mathrm{h}$ and 24 -h urinary aldosterone excretion $>12$ ug in Calhoun's studies, which was not used in this study since $24 \mathrm{~h}$ urinary aldosterone excretion was not measured. In letizia's study, a high ARR cutoff value of $40 \mathrm{ng} / \mathrm{dL}$ per $\mathrm{ng} / \mathrm{mL} / \mathrm{h}$ was considered as a detection test, and patients with PAC $>5 \mathrm{ng} / \mathrm{dL}$ after SIT was the diagnosis of PA. Nevertheless, a considerable number of people might be missed if letizia's screening criteria used not only in hypertensive males with $\mathrm{AHI}>15$ events/h but also with $\mathrm{AHI}<15$ events/h.

Patients with $15.9<\mathrm{ARR}<29.1 \mathrm{ng} / \mathrm{dL}$ per $\mathrm{ng} / \mathrm{mL} / \mathrm{h}$ were selected to discuss because ARR $<15.9 \mathrm{ng} / \mathrm{dL}$ per $\mathrm{ng} / \mathrm{mL} / \mathrm{h}$ made a diagnosis of $\mathrm{PA}$ unlikely, whereas patients with $\mathrm{ARR}>29.1 \mathrm{ng} / \mathrm{dL}$ per $\mathrm{ng} / \mathrm{mL} / \mathrm{h}$ usually may be a focus to perform the confirmatory test of PA as the same as common hypertensive patients. Work on identification of PA might be ignored when ARR ranges from 15.9 to $29.1 \mathrm{ng} / \mathrm{dL}$ per $\mathrm{ng} / \mathrm{mL} / \mathrm{h}$. From our results, we found 4 patients defined of $\mathrm{PA}$ in patients $\mathrm{AHI}>15$ events/h and $15.9<\mathrm{ARR}<29.1 \mathrm{ng} / \mathrm{dL}$ per $\mathrm{ng} / \mathrm{mL} / \mathrm{h}$. These PA patients might have been missed if uniform ARR $>29.1 \mathrm{ng} / \mathrm{dL}$ per $\mathrm{ng} / \mathrm{mL} / \mathrm{h}$ was selected as a screening criteria. More seriously, specific treatments could have been unavailable.

\section{Limitations of the study}

The present study had several limitations that may have introduced a bias. First of all, specific population was selected to this study, in which only male patients with hypertension and snoring involved. In addition, this is a retrospective observational study. Furthermore, although we followed a protocol demonstrated by guideline to screen PA, we did not use ARR as a screening criterion to investigate an optional cutoff value of ARR. And we did not use urinary aldosterone as a screening criterion because of lack of data. More importantly, identification of PA was not confirmed by pathological diagnosis, adrenalectomy or mineralcorticoid receptor therapy. In addition, clinical characteristics of snoring were not evaluated via Epworth sleepiness assessment scale. Finally, specialist bias may also have been given that all of the subjects were referred to the Hypertension Clinical Research Center.

In conclusion, this study shows that the optional cutoff values of ARR to screening for PA is different in male hypertensive patients with different severity of snoring. For patients with moderate-severe in our population, a lower cutoff value of ARR should be considered as a screening criterion.

\section{Acknowledgement}

The study is supported by grants from the National Natural Science Foundation of China (81360051) and the Foundation of People's Hospital of Xinjiang Uygur Autonomous Region (20130106). We thank Dr Tao Li from the Institute of Hypertension of the People's Hospital of Xinjiang Uygur Autonomous Region, Center of Diagnosis, Treatment and Research of Hypertension in Xinjiang, for her assistance in the endocrine monitoring

\section{References}

1. Mancia G, Fagard R, Narkiewicz K, Redon J, Zanchetti A, et al. (2014) 2013 ESH/ESC Practice Guidelines for the Management of Arterial Hypertension. Blood Press 23: 3-16.

2. Dasgupta K, Quinn RR, Zarnke KB, Rabi DM, Ravani P, et al. (2014) The 2014 Canadian Hypertension Education Program recommendations for blood pressure measurement, diagnosis, assessment of risk, prevention, and treatment of hypertension. Can J Cardiol 30: 485-501.

3. Frisoli TM, Schmieder RE, Grodzicki T, Messerli FH (2011) Beyond salt: lifestyle modifications and blood pressure. Eur Heart J 32: 3081-3087.

4. Kario K (2012) Disaster hypertension - its characteristics, mechanism, and management - . Circ J 76: 553-562.

5. Santulli G, Trimarco B, laccarino G (2013) G-protein-coupled receptor kinase 2 and hypertension: molecular insights and pathophysiological mechanisms. High Blood Press Cardiovasc Prev 20: 5-12.

6. Santulli G, Cipolletta E, Sorriento D, Del Giudice C, Anastasio A et al (2012) CaMK4 Gene Deletion Induces Hypertension. J Am Heart Assoc 1: e001081.

7. Garg N, Bhatia T, Jaiswal A (2013) Approach to a case of secondary hypertension. Clin Queries: Nephro 2: 62-70.

8. Pedrosa RP, Drager LF, Gonzaga CC, Sousa MG, de Paula LK, et al. (2011) Obstructive sleep apnea: the most common secondary cause of hypertension associated with resistant hypertension. Hypertension 58: 811-817.

9. Lüthje L, Andreas S (2008) Obstructive sleep apnea and coronary artery disease. Sleep Med Rev 12: 19-31.

10. Cintra FD, Leite RP, Storti LJ, Bittencourt LA, Poyares D, et al. (2014) Sleep Apnea and Nocturnal Cardiac Arrhythmia: A Populational Study. Arq Bras Cardiol 0.

11. Mansukhani MP, Calvin AD, Kolla BP, Brown RD Jr, Lipford MC, et al. (2013) The association between atrial fibrillation and stroke in patients with obstructive 
Citation: Wang M, Nanfang Li, Zhang Y, Zhang D, Abulikemu S, et al. (2014) Case Detection Testing for Primary Aldosteronism in Male Patients with Hypertension and Snoring. J Hypertens 3: 180. doi:10.4172/2167-1095.1000180

sleep apnea: a population-based case-control study. Sleep Med 14: 243-246.

12. Aronova A, lii TJ, Zarnegar R (2014) Management of hypertension in primary aldosteronism. World J Cardiol 6: 227-233.

13. Rossi GP, Bernini G, Caliumi C, Desideri G, Fabris B, et al. (2006) A prospective study of the prevalence of primary aldosteronism in, 125 hypertensive patients. J Am Coll Cardiol 48: 2293-2300.

14. Trifanescu R, Carsote M2, Caragheorgheopol A3, Hortopan D3, Dumitrascu A3, et al. (2013) Screening for secondary endocrine hypertension in young patients. Maedica (Buchar) 8: 108-115.

15. Hannemann A, Wallaschofski $H$ (2012) Prevalence of primary aldosteronism in patient's cohorts and in population-based studies--a review of the current literature. Horm Metab Res 44: 157-162.

16. Strauch B, Zelinka T, Hampf M, Bernhardt R, Widimsky J Jr (2003) Prevalence of primary hyperaldosteronism in moderate to severe hypertension in the Central Europe region. J Hum Hypertens 17: 349-352.

17. Stowasser M (2009) Update in primary aldosteronism. J Clin Endocrinol Metab 94: $3623-3630$

18. Inoue $Y$, Igase M, Otsuka T, Yokoyama A, Kohno N, et al. (1994) [A case report of obstructive sleep apnea syndrome associated with primary aldosteronism] Nihon Kyobu Shikkan Gakkai Zasshi 32: 288-292.

19. Calhoun DA, Nishizaka MK, Zaman MA, Harding SM (2004) Aldosterone excretion among subjects with resistant hypertension and symptoms of sleep apnea. Chest 125: 112-117.

20. Di Murro A, Petramala L, Cotesta D, Zinnamosca L, Crescenzi E, et al (2010) Renin-angiotensin-aldosterone system in patients with sleep apnoea: prevalence of primary aldosteronism. J Renin Angiotensin Aldosterone Syst 11: 165-172.

21. Narkiewicz K, Somers VK (2003) Sympathetic nerve activity in obstructive sleep apnoea. Acta Physiol Scand 177: 385-390.

22. Narkiewicz K, van de Borne PJ, Cooley RL, Dyken ME, Somers VK (1998) Sympathetic activity in obese subjects with and without obstructive sleep apnea. Circulation 98: 772-776.

23. Loredo JS, Ziegler MG, Ancoli-Israel S, Clausen JL, Dimsdale JE (1999) Relationship of arousals from sleep to sympathetic nervous system activity and BP in obstructive sleep apnea. Chest 116: 655-659.

24. Raff H, Roarty TP (1988) Renin, ACTH, and aldosterone during acute hypercapnia and hypoxia in conscious rats. Am J Physiol 254: R431-435.

25. Pratt-Ubunama MN, Nishizaka MK, Boedefeld RL, Cofield SS, Harding SM, et al. (2007) Plasma aldosterone is related to severity of obstructive sleep apnea in subjects with resistant hypertension. Chest 131: 453-459.
26. Sealey JE (1991) Plasma renin activity and plasma prorenin assays. Clin Chem 37: $1811-1819$

27. Zhang W, Si LY (2012) Obstructive sleep apnea syndrome (OSAS) and hypertension: pathogenic mechanisms and possible therapeutic approaches. Ups J Med Sci 117: 370-382.

28. Young WF (2007) Primary aldosteronism: renaissance of a syndrome. Clin Endocrinol (Oxf) 66: 607-618.

29. Funder JW, Carey RM, Fardella C, Gomez-Sanchez CE Mantero F, et al. (2008) Case detection, diagnosis, and treatment of patients with primary aldosteronism: an endocrine society clinical practice guideline. J Clin Endocrino Metab 93: 3266-3281.

30. Zhang ZH, Yu Y, Kang YM, Wei SG, Felder RB (2008) Aldosterone acts centrally to increase brain renin-angiotensin system activity and oxidative stress in normal rats. Am J Physiol Heart Circ Physiol 294: H1067-1074

31. Pimenta E, Stowasser M, Gordon RD, Harding SM, Batlouni M, et al. (2013) Increased dietary sodium is related to severity of obstructive sleep apnea in patients with resistant hypertension and hyperaldosteronism. Chest 143: 978983

32. Somers VK, White DP, Amin R, Abraham WT, Costa F, et al. (2008) Sleep apnea and cardiovascular disease: an American Heart Association/American College of Cardiology Foundation Scientific Statement from the American Heart Association Council for High Blood Pressure Research Professional Education Committee, Council on Clinical Cardiology, Stroke Council, and Council on Cardiovascular Nursing. J Am Coll Cardiol 52: 686-717.

33. Bucca CB, Brussino L, Battisti A, Mutani R, Rolla G, et al. (2007) Diuretics in obstructive sleep apnea with diastolic heart failure. Chest 132: 440-446.

34. Shiota S, Ryan CM, Chiu KL, Ruttanaumpawan P, Haight J, et al. (2007) Alterations in upper airway cross-sectional area in response to lower body positive pressure in healthy subjects. Thorax 62: 868-872.

35. Gaddam K, Pimenta E, Thomas SJ, Cofield SS, Oparil S, et al. (2010) Spironolactone reduces severity of obstructive sleep apnoea in patients with resistant hypertension: a preliminary report. J Hum Hypertens 24: 532-537.

36. Barceló A, Piérola J, Esquinas C, de la Peña M, Arqué M, et al. (2014) Relationship between Aldosterone and the Metabolic Syndrome in Patients with Obstructive Sleep Apnea Hypopnea Syndrome: Effect of Continuous Positive Airway Pressure Treatment. PLoS One 9:e84362.

37. Hsueh WA, Wyne K (2011) Renin-Angiotensin-aldosterone system in diabetes and hypertension. J Clin Hypertens (Greenwich) 13: 224-237.

38. Jansen PM, Boomsma F, van den Meiracker AH; Dutch ARRAT Investigators (2008) Aldosterone-to-renin ratio as a screening test for primary aldosteronism-the Dutch ARRAT Study. Neth J Med 66: 220-228. 